\title{
HEMATOMA HEPÁTICO SUBCAPSULAR EN SÍNDROME HELLP MANEJADAS EN EL INSTITUTO NACIONAL MATERNO PERINATAL, ENTRE LOS AÑOS 2004 A 2014.
}

\author{
Oswaldo M. Gonzales Carrillo ${ }^{1}$, Cristina D. Llanos Torres², Walter De la Peña Meniz³, \\ Giuliana C. Nalvarte Mendoza ${ }^{4}$
}

\begin{abstract}
RESUMEN
Objetivo. Determinar las características clínicas y de manejo de los casos de hematoma hepático subcapsular (HHS), como consecuencia de Preeclampsia y Síndrome HELLP durante los años 2004 al 2014. Materiales y métodos. Revisamos los casos de HHS identificados en forma retrospectiva. Se definieron las características clínicas, diagnóstico, manejo médico y quirúrgico. Identificamos también casos de la literatura mundial en PubMed. Los resultados de nuestro estudio fueron comparados con los obtenidos por la literatura mundial. Resultados. Se encontraron 25 casos de HHS. El $64 \%$ de los casos sobrevivieron y el $36 \%$ fallecieron. El $80 \%$ fueron multíparas y la ecografía abdominal fue el método diagnóstico más usado (64\%). El síntoma más frecuente fue dolor en abdomen superior (44\%), cefalea (28\%), estado de conciencia alterado $(20 \%)$ y hematuria (52\%). En el $88 \%$ se practicó empaquetamiento hepático y en el $12 \%$ observación y monitoreo. En el $50 \%$ se colocó Bolsa de Bogotá en la primera intervención quirúrgica. El promedio de estancia hospitalaria fue de 18,6 días. Conclusiones. La Incidencia de HHS fue 1 en 6000 a 8000 partos. La edad promedio fue de 33 años y la mayoría fueron multíparas. La hematuria fue un signo relevante y debiera ser motivo de estudio posterior. Se realizó empaquetamiento hepático en el $88 \%$ de los pacientes, encontrándose diferencias estadísticas con lo reportado en la literatura mundial $(p=0,0025)$ debido probablemente a la tendencia en procedimientos conservadores. A pesar de que solo usamos manejo quirúrgico, nuestras cifras de mortalidad son estadísticamente similares a la reportada en la literatura mundial $(36 \%$ vs $16 \% / p=0,08)$.
\end{abstract}

Palabras Clave: Rotura hepática; Síndrome HELLP (Fuente DeCS BIREME)

\section{HEPATIC SUBCAPSULAR HEMATOMA IN HELLP SYNDROME MANAGED BY THE MATERNAL PERINATAL NATIONAL INSTITUTE, BETWEEN 2004-2014}

\begin{abstract}
Objective. To determine the clinical and management features of hepatic sub capsular hematoma (HSH) as a result of severe preeclampsia and HELLP syndrome in the period between 2004 and 2014. Materials and methods. We reviewed the cases with $\mathrm{HSH}$ in our institution retrospectively. Clinical features, diagnosis, medical and surgical management were defined. Search also included PubMed. The results of our study were compared with those already published. Results. 25 cases of $\mathrm{HSH}$ were found. $64 \%$ of cases survived and $36 \%$ died. $80 \%$ presented in multiparous women. Abdominal ultrasound was the most commonly used diagnostic method (64\%). The most frequent symptoms were upper abdominal pain $(44 \%)$, headache $(28 \%)$, altered state of consciousness $(20 \%)$ and haematuria $(52 \%)$. In $88 \%$ of the cases, liver packing was performed and in $12 \%$ only observation and monitoring. In $50 \%$ of cases, Bogota Stock Exchange was placed during the first surgery. The average hospital stay was 18.6 days. Conclusions. The incidence of HSH was 1 in 6000 to 8000 births. The mean age was 33 years and most of them were multiparous. Haematuria was a significant sign and should be studied further. Liver packing was performed in $88 \%$ of patients, statistically different with what reported in the literature $(p=0,0025)$ likely due to the preference into conservative management. Although in our institution we used only surgical management, our mortality rate is statistically similar to that reported in the literature $(36 \%$ vs $16 \% / p=0,08)$.
\end{abstract}

Key words: Hepatic rupture; HELLP syndrome (Source: MeSH NLM)

\section{INTRODUCCIÓN}

Abercrombie, en el año 1844, fue el primero en describir la ruptura hepática como la más catastrófica complicación en el embarazo'. Poo y col, en el año 2006, realizaron una recopilación de casos de rotura hepática, encontrando 200 casos en la literatura global. La incidencia de rotura hepática se encuentra entre 1 en 45,000 y 1 en 225,000 embarazos $^{2,3}$ y ocurre aproximadamente en el 1 a $2 \%$ de los casos de preeclampsia y síndrome HELLP ${ }^{4}$. La rotura hepática, aunque es una rara complicación en el embarazo, lleva a una alta tasa de mortalidad; en algunas series puede llegar hasta el 60 a $70 \%{ }^{5}$.

La patogénesis del hematoma hepático en el síndrome HELLP no es bien conocida. Hay evidencia de un origen vascular con disfunción endotelial, lo cual produciría depósitos de fibrina, coagulación intravascular diseminada,

\footnotetext{
Médico Gíneco-Obstetra. Ex Médico Asistente del Servicio de Emergencia, Departamento de Cuidados Críticos. Jefe de la Oficina de Epidemiologia y Salud Ambiental. Instituto Nacional Materno Perinatal. Lima - Perú.

Médico Gíneco-Obstetra. Médico Asistente del Servicio de Emergencia, Departamento de Cuidados Críticos. Instituto Nacional Materno Perinatal. Lima - Perú.

3 Médico Intensivista. Jefe de la Unidad de Cuidados Intensivos Materno. Instituto Nacional Materno Perinatal. Lima - Perú.

4 Médico Residente de Gíneco-Obstetricia. Instituto Nacional Materno Perinatal. Lima - Perú.
} 


\title{
HEMATOMA HEPÁTICO SUBCAPSULAR EN SÍNDROME HELLP MANEJADAS EN EL INSTITUTO NACIONAL MATERNO PERINATAL, ENTRE LOS AÑOS 2004 A 2014.
}

\author{
Oswaldo M. Gonzales Carrillo ${ }^{1}$, Cristina D. Llanos Torres², Walter De la Peña Meniz³, \\ Giuliana C. Nalvarte Mendoza ${ }^{4}$
}

\begin{abstract}
RESUMEN
Objetivo. Determinar las características clínicas y de manejo de los casos de hematoma hepático subcapsular (HHS), como consecuencia de Preeclampsia y Síndrome HELLP durante los años 2004 al 2014. Materiales y métodos. Revisamos los casos de HHS identificados en forma retrospectiva. Se definieron las características clínicas, diagnóstico, manejo médico y quirúrgico. Identificamos también casos de la literatura mundial en PubMed. Los resultados de nuestro estudio fueron comparados con los obtenidos por la literatura mundial. Resultados. Se encontraron 25 casos de HHS. El $64 \%$ de los casos sobrevivieron y el $36 \%$ fallecieron. El $80 \%$ fueron multíparas y la ecografía abdominal fue el método diagnóstico más usado (64\%). El síntoma más frecuente fue dolor en abdomen superior (44\%), cefalea (28\%), estado de conciencia alterado $(20 \%)$ y hematuria (52\%). En el $88 \%$ se practicó empaquetamiento hepático y en el $12 \%$ observación y monitoreo. En el $50 \%$ se colocó Bolsa de Bogotá en la primera intervención quirúrgica. El promedio de estancia hospitalaria fue de 18,6 días. Conclusiones. La Incidencia de HHS fue 1 en 6000 a 8000 partos. La edad promedio fue de 33 años y la mayoría fueron multíparas. La hematuria fue un signo relevante y debiera ser motivo de estudio posterior. Se realizó empaquetamiento hepático en el $88 \%$ de los pacientes, encontrándose diferencias estadísticas con lo reportado en la literatura mundial $(p=0,0025)$ debido probablemente a la tendencia en procedimientos conservadores. A pesar de que solo usamos manejo quirúrgico, nuestras cifras de mortalidad son estadísticamente similares a la reportada en la literatura mundial $(36 \%$ vs $16 \% / p=0,08)$.
\end{abstract}

Palabras Clave: Rotura hepática; Síndrome HELLP (Fuente DeCS BIREME)

\section{HEPATIC SUBCAPSULAR HEMATOMA IN HELLP SYNDROME MANAGED BY THE MATERNAL PERINATAL NATIONAL INSTITUTE, BETWEEN 2004-2014}

\begin{abstract}
Objective. To determine the clinical and management features of hepatic sub capsular hematoma $(\mathrm{HSH})$ as a result of severe preeclampsia and HELLP syndrome in the period between 2004 and 2014. Materials and methods. We reviewed the cases with HSH in our institution retrospectively. Clinical features, diagnosis, medical and surgical management were defined. Search also included PubMed. The results of our study were compared with those already published. Results. 25 cases of $\mathrm{HSH}$ were found. $64 \%$ of cases survived and $36 \%$ died. $80 \%$ presented in multiparous women. Abdominal ultrasound was the most commonly used diagnostic method (64\%). The most frequent symptoms were upper abdominal pain $(44 \%)$, headache $(28 \%)$, altered state of consciousness $(20 \%)$ and haematuria $(52 \%)$. In $88 \%$ of the cases, liver packing was performed and in $12 \%$ only observation and monitoring. In $50 \%$ of cases, Bogota Stock Exchange was placed during the first surgery. The average hospital stay was 18.6 days. Conclusions. The incidence of HSH was 1 in 6000 to 8000 births. The mean age was 33 years and most of them were multiparous. Haematuria was a significant sign and should be studied further. Liver packing was performed in $88 \%$ of patients, statistically different with what reported in the literature $(p=0,0025)$ likely due to the preference into conservative management. Although in our institution we used only surgical management, our mortality rate is statistically similar to that reported in the literature $(36 \%$ vs $16 \% / p=0,08)$.
\end{abstract}

Key words: Hepatic rupture; HELLP syndrome (Source: MeSH NLM)

\section{INTRODUCCIÓN}

Abercrombie, en el año 1844, fue el primero en describir la ruptura hepática como la más catastrófica complicación en el embarazo'. Poo y col, en el año 2006, realizaron una recopilación de casos de rotura hepática, encontrando 200 casos en la literatura global. La incidencia de rotura hepática se encuentra entre 1 en 45,000 y 1 en 225,000 embarazos $^{2,3}$ y ocurre aproximadamente en el 1 a $2 \%$ de los casos de preeclampsia y síndrome HELLP ${ }^{4}$. La rotura hepática, aunque es una rara complicación en el embarazo, lleva a una alta tasa de mortalidad; en algunas series puede llegar hasta el 60 a $70 \%{ }^{5}$.

La patogénesis del hematoma hepático en el síndrome HELLP no es bien conocida. Hay evidencia de un origen vascular con disfunción endotelial, lo cual produciría depósitos de fibrina, coagulación intravascular diseminada,

\footnotetext{
Médico Gíneco-Obstetra. Ex Médico Asistente del Servicio de Emergencia, Departamento de Cuidados Críticos. Jefe de la Oficina de Epidemiologia y Salud Ambiental. Instituto Nacional Materno Perinatal. Lima - Perú.

Médico Gíneco-Obstetra. Médico Asistente del Servicio de Emergencia, Departamento de Cuidados Críticos. Instituto Nacional Materno Perinatal. Lima - Perú.

3 Médico Intensivista. Jefe de la Unidad de Cuidados Intensivos Materno. Instituto Nacional Materno Perinatal. Lima - Perú.

4 Médico Residente de Gíneco-Obstetricia. Instituto Nacional Materno Perinatal. Lima - Perú.
} 
hipovolemia, isquemia hepática e infartos, causando hemorragias con desarrollo de hematoma hepático subcapsular. La expansión continua del hematoma y cualquier episodio de trauma menor como palpación abdominal, transporte del paciente, contracciones uterinas, vómitos, la propia hipertensión arterial, etc.; facilitan la rotura hepática ${ }^{6-8,29}$. Así se observa, que la evaluación histopatológica del hematoma hepático revela hemorragia intraparenquimal, micro aneurismas comunes con necrosis parenquimal focal o periportal ${ }^{9}$.

El lado hepático más frecuentemente afectado es el lóbulo derecho con una frecuencia del $75 \%$, el lóbulo izquierdo en un $11 \%$ y ambos lóbulos en un $14 \%{ }^{10,27,28}$.

Los síntomas y signos clínicos son inespecíficos y va desde dolor en epigastrio o en el cuadrante superior derecho del abdomen con irradiación al hombro, hasta nauseas, vómitos y distensión abdominal. En caso de rotura del hematoma hepático, se desarrollan signos de compromiso hemodinámico ${ }^{11}$.

El ultrasonido abdominal representa el primer procedimiento útil no invasivo para diagnóstico y evaluación, es económico y de fácil acceso; sin embargo tiene limitaciones por ser operador dependiente ${ }^{27}$. La Tomografía Axial Computarizada y la Resonancia Magnética Nuclear pueden ser útiles para elucidar el diagnostico en casos ambiguos ${ }^{8}$. Frecuentemente el diagnostico se realiza al observar sangrado en los espacios parietocólicos mientras se realiza la cesárea, en una paciente con preeclampsia y síndrome HELLP ${ }^{12}$.

La morbilidad concomitante en pacientes con rotura hepática, con estancia en una Unidad de Cuidados Intensivos, incluye la coagulación intravascular diseminada en más del $90 \%$ de los casos, edema pulmonar, derrame pleural e insuficiencia renal aguda ${ }^{13}$.

La tendencia actual en el manejo del hematoma subcapsular hepático, es el manejo conservador, con monitoreo continuo, expansión de volumen y reemplazo de fluidos y transfusión sanguínea y hemoderivados. Sin embargo, la inestabilidad hemodinámica indicaría manejo quirúrgico urgente ${ }^{8}$. Se recomienda que el hematoma hepático con manejo conservador sea controlado periódicamente una vez detectado. Se han descrito casos de rotura hasta 6 semanas posteriores al diagnóstico inicial ${ }^{14,27}$

Así es que algunos autores han observado que el hematoma subcapsular hepático roto requiere laparotomía inmediata, pero que en varios casos con sangrado no activo e inclusive con signos de coagulopatía y con la paciente hemodinámicamente estable, pueden ser tratados conservadoramente ${ }^{15,16}$.

En relación al tratamiento quirúrgico, existen varios esquemas según la severidad del hematoma hepático roto y sangrante, los más utilizados son: presión con compresas de laparotomía colocadas sobre las superficies sangrantes con drenaje perihepático; la Maniobra de Pringle (comprensión digital de la arteria hepática y la vena porta por 15 ó $20 \mathrm{~min}$ ), ligadura quirúrgica del segmento hepático sangrante, sutura y drenaje, colocación de parches de epiplón y mallas quirúrgicas en la zona hepática afectada, los paquetes de material de colágeno colocados en la zona de lesión también se han utilizado y la embolización selectiva de la arteria hepática. Si la hemorragia hepática no se puede contener y se produce insuficiencia hepática aguda, se puede realizar transplante hepático. ${ }^{(13,15,29)}$. Sin embargo, si se puede controlar el sangrado exitosamente tras la laparotomía, el manejo posterior en estos pacientes se acompaña de una incidencia de sepsis postoperatoria que van desde un 20 a $30 \%{ }^{14}$.

Aun así y tras intentar múltiples procedimientos para el manejo de la rotura hepática, este sigue siendo un problema que conlleva una tasa de mortalidad muy alta. Basado en una revisión de la literatura desde los años 1960 hasta 1997, la tasa de mortalidad materna y fetal en esta revisión fue de $32 \%$ y $51 \%$ respectivamente ${ }^{12}$.

El objetivo de este estudio es recopilar los casos de hematoma subcapsular hepático roto y no roto que se han presentado en el Instituto Nacional Materno Perinatal desde el año 2004 hasta el 2014, estableciendo así la incidencia de casos presentados, las características clínicas y el manejo terapéutico recibido. Finalmente realizamos una discusión comparativa respecto a la información obtenida de nuestro estudio con los casos reportados en la literatura mundial.

\section{MATERIALES Y MÉTODOS}

Revisamos 25 casos de Hematoma Hepático Subcapsular Roto y no Roto en pacientes con Preeclampsia Severa y/o Síndrome HELLP tratados en el Instituto Nacional Materno Perinatal, entre los años 2004 a 2014. Se definió Hematoma Hepático Subcapsular, a la visualización directa del mismo a través de laparotomía y/o por medio de examen de imagen diagnóstica; en una paciente con diagnóstico de Preeclampsia Severa y/o Síndrome HELLP.

La serie de casos fue identificada en forma descriptiva y retrospectiva a través de la data proporcionada por la Unidad de Cuidados Intensivos Maternos y la Oficina de Estadística e Informática del Instituto, usando la clasificación CIE-10, indicando Hipertensión que Complica el Embarazo: Preeclampsia Severa (CIE-10 O14.1), síndrome HELLP (CIE-10 014.2) y traumatismo del hígado (CIE-10 S36.1), desde el año 2004 al 2014. En todos los casos, revisamos la información de las Historias Clínicas encontradas y aquellos casos de Preeclampsia y Síndrome HELLP con Hematoma Hepático Subcapsular, fueron separados para la obtención de información y 
llenado de una Ficha de Recolección de Datos.

Este estudio fue aprobado por el Comité de Ética del Instituto Nacional Materno Perinatal.

Se calcularon cuadros para describir las características clínicas, características de laboratorio, forma de diagnóstico, manejo médico y manejo quirúrgico establecido, así como las complicaciones posteriores al manejo.

Identificamos también casos de la literatura mundial, realizando búsqueda en la base de datos de PubMed, entre los años 2004 y 2014 identificando artículos relevantes. La búsqueda fue limitada a artículos en Inglés, también se incluyeron resúmenes de artículos que permitían información requerida. La búsqueda fue realizada usando los términos $\mathrm{MeSH}$ "hepatic rupture" y "HELLP síndrome", establecimos una lista de artículos primarios y revisiones, finalmente realizamos un cuadro resumen con datos importantes.

Los resultados de nuestro estudio fueron comparados con los obtenidos por la literatura mundial, usando medias para variables continuas normalmente distribuidas y proporciones para variables categóricas o binarias. Las diferencias entre nuestra serie de casos y los casos obtenidos por la literatura mundial fueron comparadas usando el test exacto de Fisher para variables categóricas y el test de resumen de rangos de Wilcoxon para variables continuas. El análisis estadístico fue realizado usando STATA 11.

\section{RESULTADOS}

Los casos de Hematoma Hepático Subcapsular encontrados en nuestro estudio fueron 30 , de los cuales solo se encontraron 25 casos con registros completos. Observamos que la media de edad fue de 33 años (rango de 18 a 48 años de edad).

En la siguiente tabla se observa la cantidad de casos, presentados por años, desde el 2004 al 2014, observándose un aumento de casos en el último año (6 casos).

Del total de casos presentados, el $64 \%$ (17 pacientes) sobrevivieron y el $36 \%$ (8 pacientes) fallecieron.

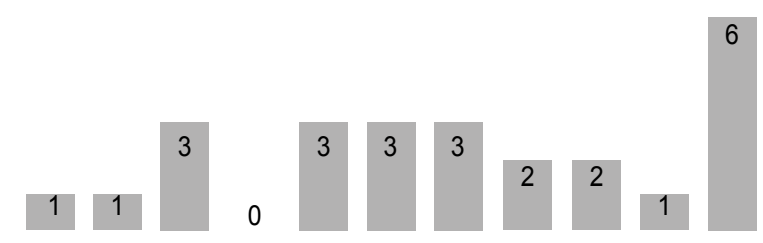

20042005200620072008200920102011201220132014

Gráfico 1. Número de Casos de Hematoma Hepático Subcapsular. 2004 al 2014 - INMP

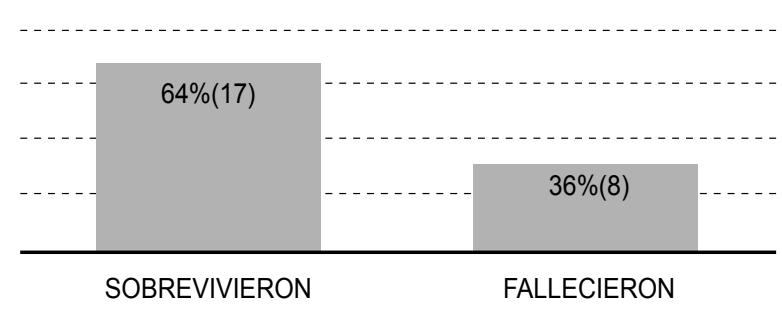

Gráfico 2. Sobrevida en Pacientes con Hematoma Hepático Subcapsular. 2004-2014.

Todas las pacientes fueron referidas de los Distritos de Lima (56\%), en segundo lugar desde Ancash (16\%) y en tercer lugar Huancavelica (12\%).

En la siguiente tabla observamos que el $20 \%$ de las pacientes con rotura hepática fueron nulíparas y el $80 \%$ multíparas, el $40 \%$ de las pacientes ingresó por emergencia al instituto como gestante y el $60 \%$ como puérpera, que la ecografía abdominal fue el método diagnóstico más frecuente para rotura hepática $(64 \%)$ frente a un $36 \%$ de pacientes que fueron diagnosticados durante la cesárea. Finalmente, de las pacientes que fueron referidas en el instituto, $88 \%$ terminaron por cesárea o CesareaHisterectomia y $12 \%$ terminaron en parto eutócico.

El síntoma más frecuente observado fue dolor en abdomen superior (44\%) seguido de cefalea (28\%). Un grupo de pacientes $(20 \%)$ observados en la tabla, se refieren a las pacientes que llegaron con estado de conciencia alterado por emergencia y no pudo definirse la sintomatología presentada. Un dato importante es el referido a la presencia de Hematuria Macroscópica observada durante la evaluación y corroborada con el examen de laboratorio que llega a un $52 \%$ de los casos presentados.

Tabla 1. Características Generales de las pacientes con Hematoma Hepático Subcapsular

\begin{tabular}{lcc}
\hline Características Generales & $\mathbf{N}^{\circ}$ & $\%$ \\
\hline Paridad & 5 & 20 \\
Nulíparas & 20 & 80 \\
\hline Multíparas & & \\
Condición Obstétrica & 10 & 40 \\
\hline Gestante & 15 & 60 \\
\hline Puérperas & & \\
\hline Forma de Diagnóstico de Ruptura Hepática & 16 & 64 \\
\hline Diagnóstico Ecográfico & 9 & 36 \\
\hline Diagnóstico en Cesárea & & \\
Diagnóstico 1 de Ingreso & 10 & 40 \\
\hline Gestante & 15 & 60 \\
\hline Puérpera post cesárea o parto vaginal & & \\
\hline Diagnóstico 2 de Ingreso & 9 & 36 \\
\hline Pre eclampsia severa & 16 & 64 \\
\hline PE + Síndrome HELLP & & \\
\hline Término de Embarazo & 3 & 12 \\
\hline Parto Eutócico & 16 & 64 \\
\hline Cesárea & 6 & 24 \\
\hline Cesarea-Histerectomia &
\end{tabular}


Tabla 2. Síntomas principales en las pacientes con Hematoma Hepático Subcapsular

\begin{tabular}{lcc}
\hline \multicolumn{1}{c}{ Síntomas } & $\mathbf{N}^{\circ}$ & $\%$ \\
\hline dolor abdomen superior & 11 & 44 \\
cefalea & 7 & 28 \\
estado de conciencia alterado* & 5 & 20 \\
escotomas visuales & 1 & 4 \\
tinnitus & 1 & 4 \\
hematuria & 13 & 52 \\
\hline
\end{tabular}

En la siguiente tabla se muestran los valores de laboratorio observados, definiéndose la media de valores y los valores mínimos y máximos de cada examen de laboratorio obtenido. La media de Hemoglobina fue de $9.1 \mathrm{mg} / \mathrm{dL}$, con valores tan bajos de hasta $3.3 \mathrm{mg} / \mathrm{dL}$. Hubo casos de pacientes con hipoglicemia, con valores de hasta $55 \mathrm{gr} /$ dL. Valores de Transaminasas y Bilirrubinas altas llaman también la atención, llegando a valores de transaminasas mayores a $2000 \mathrm{U} / \mathrm{L}$ y de Bilirrubinas totales mayores de $14 \mathrm{mg} / \mathrm{dL}$.

Tabla 3. Resultados de Laboratorio en pacientes con Hematoma Hepático Subcapsular

\begin{tabular}{lccc}
\hline Exámenes de Laboratorio & Media & $\begin{array}{c}\text { Valor } \\
\text { Mínimo }\end{array}$ & $\begin{array}{c}\text { Váor } \\
\text { Máximo }\end{array}$ \\
\hline Hemoglobina (gr/dL) & 9.1 & 3.3 & 13.6 \\
Plaquetas (pmc) & 108,240 & 16,000 & 250,000 \\
TP $(\mathrm{seg})$ & 18.8 & 10 & 54 \\
TPTa (seg) & 55.4 & 26.4 & 292 \\
Glucosa (gr/dL) & 106.6 & 55 & 190 \\
Creatinina (gr/dL) & 1.4 & 0.54 & 3.5 \\
TGO (U/L) & 681.6 & 30 & 2860 \\
TGP (U/L) & 657.2 & 18 & 2410 \\
Bilirrubinas Totales (gr/dL) & 2.96 & 0.43 & 14.5 \\
Bilirrubinas Directas $(\mathrm{gr} / \mathrm{dL})$ & 1.2 & 0.18 & 7.2 \\
Bilirrubinas Indirectas $(\mathrm{gr} / \mathrm{dL})$ & 1.75 & 0.18 & 10.25 \\
\hline
\end{tabular}

El uso de Metildopa y Sulfato de Magnesio se reporta en el $56 \%$ y $72 \%$ de los casos respectivamente. El uso de Inotrópicos se reportó en el $32 \%$, el uso de diálisis en el $36 \%$, y el uso ventilación mecánica en el $64 \%$. Hasta en el $92 \%$ de las pacientes se usó hemoderivados para la estabilización hemodinámica.

Tabla 4. Tratamiento médico en pacientes con Hematoma Hepático Subcapsular

\begin{tabular}{lcc}
\hline \multicolumn{1}{c}{ Tratamiento Médico Recibido } & $\mathbf{N}^{\circ}$ & $\%$ \\
\hline Metildopa & 14 & 56 \\
Sulfato de Magnesio & 18 & 72 \\
Inotrópicos & 8 & 32 \\
Transfusión de Paq. Globulares & 23 & 92 \\
Transfusión de Plaquetas & 21 & 84 \\
\hline Transfusión de PFC & 18 & 72 \\
Transfusión de Crioprecipitados & 13 & 52 \\
Uso Diálisis & 9 & 36 \\
Ventilación Mecánica & 16 & 64 \\
\hline
\end{tabular}

Tabla 5. Número de hemoderivados en pacientes con Hematoma Subcapsular Hepático

\begin{tabular}{lccc}
\hline Paquetes Sang. Administrados & Media & Mínimo & Máximo \\
\hline Nro. Paquetes Globulares & 7.4 & 0 & 28 \\
Nro. Plaquetas & 10.3 & 0 & 76 \\
Nro. PFC & 10.3 & 0 & 60 \\
Nro. Crioprecipitados & 4.6 & 0 & 42 \\
\hline
\end{tabular}

Una cantidad importante de hemoderivados fueron usados en las pacientes, la media de paquetes globulares usados fue de 7.4 por paciente, con un máximo de 28 en una paciente, la media de bolsas de Plaquetas y Plasma Fresco Congelado usados fue de 10.3 bolsas por paciente con un máximo de 76 y 60 respectivamente. Finalmente la media de Crioprecipitados usados fue de 4.6 por paciente con un máximo de 42 paquetes en una paciente.

Respecto al manejo quirúrgico, en 22 pacientes (88\%) se identificó Hematoma Hepático Subcapsular roto y se les practicó Empaquetamiento Hepático con compresas para la hemostasia del sangrado; 3 pacientes (12\%) tuvieron Hematoma Hepático Subcapsular no roto, a quienes se les realizó solo Observación y Monitoreo. A estas últimas se les observó el hematoma hepático por laparotomía y no se realizó intervención hepática directa. Cabe señalar que solo en el 50\% (11 casos con Hematoma Hepático Subcapsular roto) se realizó la colocación de Bolsa de Bogotá en la primera intervención quirúrgica. De las pacientes manejadas con empaquetamiento hepático, se usó además Argón Plasma en 2 de ellas y Malla de Colágeno en otra.

Tabla 6. Tratamiento quirúrgico en las pacientes con Hematoma Hepático Subcapsular

\begin{tabular}{|c|c|c|}
\hline Hematoma Hepático Subcapsular & $\mathbf{N}^{\circ}$ & $\%$ \\
\hline Roto & 22 & 88 \\
\hline No Roto & 3 & 12 \\
\hline $\begin{array}{l}\text { Tratamiento Quirúrgico en Hematoma } \\
\text { Subcapsular Roto }\end{array}$ & $\mathbf{N}^{\circ}$ & $\%$ \\
\hline Empaquetamiento Hepático (EH) & 22 & 100 \\
\hline - EH + Uso Argón - Plasma & 2 & 10 \\
\hline - EH + Uso Malla de Colágeno & 1 & 5 \\
\hline - Uso de Bolsa de Bogotá primera intervención & 11 & 50 \\
\hline
\end{tabular}

El $100 \%$ de las pacientes que tuvieron hematoma hepático subcapsular roto fueron reintervenidas, la media de laparotomías por pacientes fue 2 , con valor máximo de 5 relaparotomías en 1 paciente.

Las complicaciones más frecuentes observadas fueron Insuficiencia Renal Aguda y Falla Orgánica Múltiple (40\% de los casos para cada complicación), seguido por Shock Hipovolémico (32\% de los casos), Injuria Pulmonar, CID e Infección de Herida Operatoria (24\% para cada una); finalmente Sepsis - Shock Séptico ( $20 \%$ de los casos) y otros, como se observa en el siguiente cuadro. 
Tabla 7. Complicaciones post quirúrgicas en Hematoma Subcapsular Hepático

\begin{tabular}{lcc}
\hline \multicolumn{1}{c}{ Complicaciones Post Cirugía } & $\mathbf{N}^{\circ}$ & $\%$ \\
\hline Insuficiencia Renal Aguda & 10 & 40 \\
Falla Orgánica Múltiple & 10 & 40 \\
Shock Hipovolémico & 8 & 32 \\
Injuria Pulmonar & 6 & 24 \\
Coagulación Intravascular Diseminada & 6 & 24 \\
Infección de Herida Operatoria & 6 & 24 \\
Sepsis- Shock Séptico & 5 & 20 \\
Eventración & 3 & 12 \\
Hipertensión Intra abdominal & 2 & 8 \\
Neumonía Intrahospitalaria & 2 & 8 \\
\hline
\end{tabular}

El promedio de días de estancia hospitalaria fue de 18.6, siendo el valor mínimo de 1 día y el máximo de 46 días.

\section{DISCUSIÓN}

Los casos de rotura hepática en el embarazo son raros, con un rango de incidencia reportada de 1 en 45 mil a 1 en 225 mil partos ${ }^{9}$. En nuestro instituto el promedio de partos es de 15 mil a 20 mil anualmente, lo que hace que la incidencia de rotura hepática sea de 1 en 6000 a 1 en 8000 partos, considerando que somos un centro de referencia nacional. No hemos encontrado otras publicaciones de casos de rotura hepática en nuestro país.

Al revisar la literatura mundial, encontramos 47 estudios de reportes de casos de Rotura Hepática, estos estudios reportan desde 1 a 10 pacientes en su serie de casos. En nuestro estudio el número de casos reportados en 10 años fue de 25 pacientes, siendo hasta el momento la serie de casos más amplia publicada en este tema.

Tabla 9. Artículos y revisiones encontradas en la búsqueda de la literatura entre los años 2004 al 2014

\begin{tabular}{|c|c|c|c|c|c|c|c|c|}
\hline & $\begin{array}{c}\text { Edad } \\
\text { (años) }\end{array}$ & Paridad & $\begin{array}{c}\text { Edad } \\
\text { Gestacional }\end{array}$ & Preeclampsia & Tratamiento & $\begin{array}{l}\text { Tipo de } \\
\text { Parto }\end{array}$ & $\begin{array}{c}\text { Muerte } \\
\text { Materna }\end{array}$ & $\begin{array}{c}\text { Muerte } \\
\text { Fetal }\end{array}$ \\
\hline Kanchana et al 2012 & 26 & Primípara & 34 & si & Observación & $?$ & si & si \\
\hline \multirow{8}{*}{ El Youssoufi et al 2007} & 41 & Multípara & 34 & si & Quirúrgico & Cesárea & si & si \\
\hline & 22 & Primípara & 35 & si & Observación & Cesárea & no & no \\
\hline & 26 & Multípara & 38 & si & Quirúrgico & Vaginal & si & no \\
\hline & 40 & Multípara & 29 & si & Quirúrgico & Cesárea & no & si \\
\hline & 35 & Multípara & 34 & si & Quirúrgico & Cesárea & no & no \\
\hline & 27 & Multípara & 28 & si & Observación & Vaginal & si & si \\
\hline & 35 & Multípara & 25 & si & Quirúrgico & Cesárea & si & si \\
\hline & 25 & Primípara & 37 & si & Quirúrgico & Cesárea & si & no \\
\hline Kelly et al 2009 & 35 & Primípara & 18 & $?$ & Quirúrgico & Cesárea & no & si \\
\hline \multirow[t]{3}{*}{ Pavlis et al 2009} & 38 & $?$ & 38 & si & Quirúrgico & Cesárea & no & no \\
\hline & 38 & $?$ & 37 & $?$ & Laser Argón & Cesárea & no & no \\
\hline & 32 & $?$ & $?$ & $?$ & Qx y Factor VII & Cesárea & no & no \\
\hline Miguelote et al 2009 & 38 & Primípara & 36 & si & Quirúrgico & Cesárea & no & no \\
\hline Raga et al 2008 & 31 & Primípara & 36 & no & $?$ & Cesárea & no & no \\
\hline \multirow[t]{3}{*}{ Nunes et al 2005} & 38 & Primípara & 31 & si & $\begin{array}{l}\text { Qx y } \\
\text { bolización }\end{array}$ & Cesárea & no & si \\
\hline & 33 & Multípara & 33 & si & Observación & Cesárea & no & no \\
\hline & 27 & Multípara & 30 & no & Quirúrgico & Cesárea & si & si \\
\hline Dart et al 2004 & 24 & Multípara & 22 & si & Factor VII & Vaginal & si & si \\
\hline \multirow{3}{*}{ Merchant et al 2004} & 30 & Multípara & 23 & si & Qx y Factor VII & Cesárea & si & si \\
\hline & $<30$ & Primípara & 32 & si & Qx y Factor VII & Cesárea & no & si \\
\hline & 30 & Primípara & 33 & si & Factor VII & Cesárea & no & si \\
\hline \multirow{3}{*}{$\begin{array}{l}\text { Shrivastava et al } 2006 \\
\text { Descheemaeker et al } \\
2006 \\
\text { Gilboa et al } 2006\end{array}$} & $?$ & $?$ & 33 & si & \multirow{2}{*}{$\begin{array}{l}\text { Laser Argón } \\
\text { Qx y Factor VII y } \\
\text { Laser Argón }\end{array}$} & Cesárea & no & no \\
\hline & 33 & Multípara & 39 & si & & Vaginal & no & no \\
\hline & 25 & Primípara & 35 & si & Observación & Cesárea & no & no \\
\hline Srivastava et al 2007 & 31 & Primípara & 27 & si & $\begin{array}{c}\text { Qx y } \\
\text { Embolización }\end{array}$ & Cesárea & no & si \\
\hline \multirow{6}{*}{$\begin{array}{l}\text { Dessole et al } 2007 \\
\text { Wicke et al } 2004\end{array}$} & 35 & Primípara & 38 & si & Quirúrgico & Cesárea & no & no \\
\hline & 33 & Multípara & 37 & no & Observación & Cesárea & no & no \\
\hline & 26 & Primípara & 36 & no & Observación & Cesárea & no & no \\
\hline & 26 & Primípara & 22 & si & Observación & Vaginal & no & si \\
\hline & 30 & Multípara & 40 & no & Quirúrgico & Cesárea & no & $?$ \\
\hline & 29 & Primípara & 34 & no & Transplante & Cesárea & no & ? \\
\hline Sutton et al 2008 & 25 & Multípara & $?$ & no & Observación & Cesárea & si & no \\
\hline \multirow{2}{*}{$\begin{array}{l}\text { Chou et al } 2010 \\
\text { Shaw et al } 2005\end{array}$} & 33 & Multípara & 26 & $?$ & Quirúrgico & Cesárea & no & si \\
\hline & 39 & Multípara & 40 & no & Observación & Vaginal & no & no \\
\hline Harris et al 2005 & 24 & Primípara & 32 & si & $\begin{array}{c}\text { Qx y } \\
\text { Embolización y } \\
\text { laser argón }\end{array}$ & Cesárea & no & no \\
\hline \multirow{11}{*}{$\begin{array}{l}\text { Wust et al } 2004 \\
\text { Gutiérrez et al } 2010 \\
\text { Mikou et al } 2008 \\
\text { Varotti et al } 2010 \\
\text { Quesnel et al } 2012 \\
\text { Araujo et al } 2006\end{array}$} & 31 & Primípara & 27 & si & Quirúrgico & Cesárea & no & si \\
\hline & 25 & $?$ & 39 & $?$ & Quirúrgico & Cesárea & no & no \\
\hline & 31 & Multípara & 39 & si & Quirúrgico & Cesárea & no & no \\
\hline & 43 & Multípara & 38 & si & Transplante & Cesárea & no & no \\
\hline & 28 & Primípara & 32 & si & Qx y Factor VII & Cesárea & no & no \\
\hline & 36 & Multípara & 36 & si & Quirúrgico & Cesárea & no & si \\
\hline & 44 & Multípara & $?$ & si & Observación & Cesárea & si & si \\
\hline & 43 & Multípara & 24 & si & Quirúrgico & Cesárea & no & si \\
\hline & 48 & Multípara & $?$ & si & Quirúrgico & Cesárea & no & si \\
\hline & 43 & Multípara & 37 & si & Quirúrgico & Cesárea & no & si \\
\hline & 42 & Multípara & 34 & si & Quirúrgico & Cesárea & no & si \\
\hline
\end{tabular}




\begin{tabular}{|c|c|c|c|c|c|c|c|c|}
\hline & $\begin{array}{l}\text { Edad } \\
\text { (años) }\end{array}$ & Paridad & $\begin{array}{c}\text { Edad } \\
\text { Gestacional }\end{array}$ & Preeclampsia & Tratamiento & $\begin{array}{c}\text { Tipo de } \\
\text { Parto }\end{array}$ & $\begin{array}{c}\text { Muerte } \\
\text { Materna }\end{array}$ & $\begin{array}{c}\text { Muerte } \\
\text { Fetal }\end{array}$ \\
\hline & 45 & Multípara & 40 & si & Quirúrgico & Vaginal & no & si \\
\hline & 42 & Multípara & 35 & si & Quirúrgico & Vaginal & no & si \\
\hline & 38 & Multípara & 38 & si & Observación & Vaginal & no & no \\
\hline & 27 & Multípara & 33 & si & Quirúrgico & Vaginal & no & no \\
\hline Stella et al 2008 & 33 & Primípara & 31 & no & Laser Argón & Cesárea & no & no \\
\hline Seren et al 2006 & 32 & Primípara & 37 & si & Quirúrgico & Cesárea & no & no \\
\hline Shames et al 2005 & 37 & Multípara & 35 & si & Transplante & Vaginal & no & no \\
\hline Carlson et al 2004 & 27 & Primípara & 34 & si & Observación & Cesárea & no & $?$ \\
\hline Maoz et al 2010 & 27 & $?$ & $?$ & $?$ & Observación & $?$ & no & $?$ \\
\hline Zeirideen et al 2009 & 28 & Primípara & 35 & si & Quirúrgico & Cesárea & no & no \\
\hline \multirow{3}{*}{ Wilson et al 2014} & 38 & $?$ & 29 & si & Quirúrgico & Cesárea & no & no \\
\hline & 34 & Primípara & 34 & si & Observación & Cesárea & no & no \\
\hline & 32 & Primípara & 31 & si & Qx y Factor VII & Cesárea & no & si \\
\hline Das et al 2007 & 22 & Primípara & 36 & $?$ & $\begin{array}{c}\text { Qx y } \\
\text { Embolización }\end{array}$ & Cesárea & no & $?$ \\
\hline Connor et al 2009 & 28 & Primípara & 37 & si & Transplante & Cesárea & no & no \\
\hline Hafeez et al 2005 & 29 & Multípara & 28 & si & Observación & Cesárea & no & si \\
\hline Singh et al 2009 & 22 & Multípara & 34 & si & Quirúrgico & Cesárea & si & si \\
\hline Fat et al 2011 & 24 & Multípara & 38 & no & Quirúrgico & Cesárea & no & no \\
\hline \multirow[t]{8}{*}{ Zarrinpar et al 2007} & 34 & $?$ & $?$ & $?$ & Transplante & Vaginal & no & no \\
\hline & 25 & $?$ & $?$ & $?$ & Transplante & Cesárea & si & no \\
\hline & 25 & $?$ & $?$ & $?$ & Transplante & Vaginal & no & no \\
\hline & 33 & $?$ & $?$ & $?$ & Transplante & $?$ & no & si \\
\hline & 27 & ? & ? & $?$ & Transplante & Cesárea & no & no \\
\hline & 23 & $?$ & $?$ & $?$ & Transplante & Cesárea & no & no \\
\hline & 37 & $?$ & $?$ & $?$ & Transplante & Cesárea & si & no \\
\hline & 32 & $?$ & ? & $?$ & Transplante & Cesárea & no & si \\
\hline Grand'Matson et al & 32 & Primípara & 30 & si & Embolización & Cesárea & no & si \\
\hline \multirow[t]{8}{*}{2012} & 29 & Primípara & 36 & si & Embolización & Cesárea & no & no \\
\hline & 28 & Primípara & 36 & si & Embolización & Cesárea & no & no \\
\hline & 23 & Multípara & 32 & si & $\begin{array}{c}\text { Qx y } \\
\text { Embolización }\end{array}$ & Cesárea & no & no \\
\hline & 27 & Primípara & 22 & si & Quirúrgico & Vaginal & no & si \\
\hline & 29 & Primípara & 40 & si & Embolización & Cesárea & no & no \\
\hline & 27 & Multípara & 35 & si & Embolización & Vaginal & no & no \\
\hline & 32 & Multípara & 35 & si & Quirúrgico & Vaginal & no & no \\
\hline & 35 & Multípara & 25 & si & Observación & Cesárea & no & si \\
\hline
\end{tabular}

\begin{tabular}{|c|c|c|c|c|c|c|c|c|}
\hline \multicolumn{9}{|c|}{ Artículos con resúmenes en inglés pero escritos en otro lenguaje, los datos son solo de los resúmenes. } \\
\hline Suner et al 2009 & 41 & $?$ & 39 & si & ? & $?$ & no & ? \\
\hline Martinez et al 2010 & $?$ & ? & $?$ & $?$ & Quirúrgico & Cesárea & $?$ & $?$ \\
\hline Millan et al 2010 & ? & Multípara & $?$ & no & Quirúrgico & $?$ & no & no \\
\hline \multirow[t]{4}{*}{ Pliego et al 2006} & 24 & Multípara & 38 & si & Quirúrgico & Cesárea & si & no \\
\hline & 41 & Multípara & 36 & si & Quirúrgico & Cesárea & no & si \\
\hline & 30 & Multípara & 37 & si & Observación & Cesárea & no & no \\
\hline & 26 & Multípara & 37 & si & $\begin{array}{c}\text { Qx y } \\
\text { Embolización }\end{array}$ & Cesárea & no & no \\
\hline \multirow[t]{3}{*}{ Boormans et al 2007} & 33 & $?$ & $?$ & si & Quirúrgico & Cesárea & no & no \\
\hline & 33 & $?$ & $?$ & si & Quirúrgico & Cesárea & no & si \\
\hline & 35 & $?$ & $?$ & si & Quirúrgico & Cesárea & no & no \\
\hline \multirow{3}{*}{$\begin{array}{l}\text { You et al } 2014 \\
\text { Descheemaeker et al } \\
2009 \\
\text { Miguelote et al } 2009\end{array}$} & 30 & Primípara & $?$ & si & $?$ & $?$ & $?$ & $?$ \\
\hline & $?$ & $?$ & $?$ & si & Transplante & $?$ & $?$ & $?$ \\
\hline & 28 & Primípara & 36 & si & Quirúrgico & Cesárea & no & $?$ \\
\hline
\end{tabular}

* "Tratamiento Quirúrgico" incluye: Empaquetamiento, ligadura de la arteria hepática, hemihepatectomia y cirugía no especificada

Nosotros reportamos que la edad promedio en las pacientes fue de 33 años, siendo similar a la literatura mundial, en la que se observa una edad promedio de 32 años (tabla 10). Solo Grand'Maison et al, en su serie de 9 casos en 10 años realizado en Canadá en el 2012, reporta una edad promedio menor de 30 años ${ }^{12}$. Esto podría explicarse por diferencias socio-ambientales que creemos pueden ser materia de estudio.

La mayoría de pacientes en nuestro estudio fueron multíparas $(80 \%)$, como también se observa en los estudios reportados mundialmente (55\%).

En relación a la fisiopatología, el aumento de flujo sanguíneo en los órganos durante el embarazo y la alteración endotelial predispone a la formación de
Tabla 10. Comparación de resultados encontrados en el INMP y la literatura mundial.

\begin{tabular}{lccc}
\hline \multicolumn{1}{c}{ Variable } & $\begin{array}{c}\text { Casos del } \\
\text { Estudio } \\
(\mathbf{N = 2 5})\end{array}$ & $\begin{array}{c}\text { Casos de la } \\
\text { Literatura* } \\
(\mathbf{N = 9 5 )}\end{array}$ & $\boldsymbol{p}$ \\
\hline Edad Materna & $33(18-48)$ & $32(22-48)$ & 0.2359 \\
Multíparas & $20 / 25(80 \%)$ & $41 / 74(55 \%)$ & 0.03 \\
Parto por cesárea & $22 / 25(88 \%)$ & $72 / 88(82 \%)$ & 0.56 \\
Manejo solo con Cirugía & $22 / 25(88 \%)$ & $51 / 92(55 \%)$ & 0.0025 \\
Manejo solo con & $3 / 25(12 \%)$ & $18 / 92(19 \%)$ & 0.56 \\
Observación & $8 / 25(36 \%)$ & $15 / 92(16 \%)$ & 0.08 \\
\hline Mortalidad Materna & & &
\end{tabular}

Los datos están definidos en media (edad mínima - edad máxima) y $\mathrm{n} / \mathrm{N}(\%)$ Los valores de "p" son obtenidos usando la prueba de Wilcoxon para la edad y test exacto de Fisher para las demás variables.

* Los valores totales de los casos obtenidos de la literatura son diferentes debido a datos no encontrados 
hematomas hepáticos. En la presentación clínica de la rotura hepática, las pacientes pueden tener síntomas de duración variable como: Cefalea, dolor en epigastrio y/o en cuadrante superior derecho, hipotensión sistémica, signos de irritación peritoneal, hepatomegalia y anemia ${ }^{31}$. En nuestro estudio la principal sintomatología clínica reportada fue el dolor en abdomen superior (44\%), seguido por cefalea $(28 \%)$, escotomas visuales y tinnitus ( $4 \%$ cada una). En el $20 \%$ de nuestros casos no se pudo definir la característica clínica principal debido al mal estado general en el que llegaron las pacientes a nuestro instituto. Similar a nuestro estudio, Rinehart et al (22) reportó como principal sintomatología la epigastralgia $(70 \%)$, seguido de hipertensión (66\%), shock (56\%), náuseas y vómitos $(25 \%)$, dolor en hombro $(21 \%)$ y cefalea $(11 \%)$. Un dato importante hallado en nuestro reporte de casos es el referido a la presencia de hematuria en un gran número $(52 \%)$, no encontrando este signo en ningún otro estudio reportado en la literatura mundial y que en nuestra serie de casos fue relevante y debiera ser motivo de estudio posterior.

Los resultados de laboratorio revelan alteraciones muy marcadas de la función hepática y de la coagulación. Podemos observar en nuestro estudio valores de transaminasas tan altos como hasta de $2000 \mathrm{U} / \mathrm{L}$, valores de bilirrubinas totales de hasta $14.5 \mathrm{mg} / \mathrm{dL}$, valores muy alterados en recuento de plaquetas y tiempos de coagulación, además de casos de hipoglicemia marcada de hasta $55 \mathrm{mg} / \mathrm{dL}$. Estos datos nos llevan a pensar que algunos pacientes con estos valores tan alterados, además de rotura hepática, pueden haber estado complicados con hígado graso agudo del embarazo ${ }^{33}$. Estos hallazgos ameritan estudio posterior.

La ecografía abdominal y la Tomografía Axial Computarizada abdominal son los métodos de elección en la confirmación del diagnóstico si la paciente está estable $^{12}$. En nuestro estudio el diagnostico se realizó con ecografía abdominal en un $64 \%$ de los casos. Los hallazgos encontrados durante la ecografía fueron presencia de líquido libre en cavidad abdominal (hemoperitoneo) y en otros la evidencia del hematoma hepático subcapsular no roto. En el $36 \%$ restante, el diagnóstico fue realizado inadvertidamente con visualización directa del hematoma hepático y/o hemoperitoneo durante la cesárea.

El manejo adecuado de la lesión hepática incluye desde manejo conservador con terapia de las complicaciones hasta el manejo quirúrgico en combinación con terapia de soporte que incluye infusión de líquidos, sangre y hemoderivados. En nuestros casos reportados el promedio de transfusiones fue de 7.4 paquetes globulares por paciente, 10.3 unidades de Plaquetas, 10.3 Unidades de Plasma fresco Congelado y 4.6 Unidades de Crioprecipitados. Pliego Pérez et al, en el estudio de 4 casos reportados con rotura hepática en México en el año 2006, publica la utilización de hasta 80 hemoderivados en una de las pacientes de su estudio ${ }^{30}$. Nosotros hemos encontrado la utilización de hasta 206 hemoderivados en una sola paciente con rotura hepática, la cual fue referida de Huánuco a nuestro instituto en mal estado general.

Si bien el manejo conservador en casos de hematoma hepático no complicado está claramente establecido con la observación y seguimiento por estudios de imagen, no hay pautas claras respecto a cuál es la mejor estrategia en casos de rotura hepático-subcapsular ${ }^{28}$. En nuestro estudio, el manejo conservador solo con monitoreo y observación de las pacientes fue de $12 \%$, la cual no muestra diferencias estadísticamente significativas con los hallazgos encontrados en la literatura mundial, el cual fue $19 \%(p=0.56)$.

El manejo quirúrgico varía desde la reparación del sitio de la lesión, empaquetamiento, ligadura de la arteria hepática, embolización de la arteria hepática, hasta el trasplante ${ }^{28}$. En nuestro estudio se realizó manejo quirúrgico con empaquetamiento hepático en el $88 \%$ de los casos. Estos resultados muestran diferencias estadísticamente significativas con lo reportado en la literatura mundial $(p$ $=0.0025$ ). Aunque en los estudios revisados el porcentaje de manejo quirúrgico es más de la mitad de los casos $(55 \%)$, este manejo es menor en relación a lo observado en nuestra serie de casos (88\%).

Solo en el $50 \%$ de las pacientes con rotura hepática se colocó Bolsa de Bogotá ${ }^{34}$ en la primera intervención, sin embargo 2 de ellas tuvieron hipertensión intraabdominal persistente. Desde el año 2014 en el instituto, además del uso de bolsa de Bogotá en las pacientes intervenidas quirúrgicamente para prevenir el síndrome compartamental, el uso del sistema de presión negativa con vacuum pack ${ }^{35}$ en estas pacientes ha mejorado el pronóstico y la estancia hospitalaria.

Si bien la laparotomía de emergencia ha sido el manejo de elección para las pacientes con hematoma hepático subcapsular roto, los últimos reportes proponen como estrategia de manejo en este tipo de pacientes la angiografía con embolización selectiva de la arteria hepática, previa resucitación hemodinámica de la paciente, seguida de laparotomía de emergencia con manejo quirúrgico en caso de inestabilidad persistente a pesar de la embolización ${ }^{14}$, procedimiento que aún no realizamos en nuestro instituto.

La mortalidad materna observada en los casos revisados de la literatura es del $16 \%$, comparado con nuestra serie de casos $(36 \%)$, aunque porcentualmente encontremos una diferencia de más del doble, no existe una diferencia estadísticamente significativa $(p=0,08)$. Consideramos que a pesar de que solo usamos manejo quirúrgico con empaquetamiento hepático, nuestras cifras de mortalidad materna son estadísticamente similares a la reportada en la literatura mundial. 


\section{REFERENCIAS BIBLIOGRÁFICAS}

1. Abercrombie J. Case of hemorrhage of the liver. London Medical Gazzette 1844;34:72.

2. Poo JL, Gongora J. Hepatic haematoma and hepatic rupture in pregnancy. Annals of Hepatology 2006, 5(3):224-226.

3. Sibai BM, Ramadan MK, Usta I, Salama M, Mercer BM, Friedman SA. Maternal morbidity and mortality in 442 pregnancies with hemolysis elevated liver enzymes and low platelets (HELLP syndrome). Am J Obstet Gynecol 1993; 169:1000-6

4. van Dyke RW. The liver in pregnancy in Hepatology: A Text Book of Liver Disease, pp. 1408-1418, 2nd edition, 1990.

5. Mushambi MC, Halligan AW. Recent development in the pathophysiology and the management of pre-eclampsia. British Journal of Anaesthesia 1996: 76: 133-148.

6. Araujo A. Characteristics and treatment of hepatic rupture caused by HELLP syndrome. Am J Obstet Gynecol 2006; 195: 129-33.

7. Dessole S, Capobianco G, Virdis P, Rubattu G, Cosmi E, and Porcu A. Hepatic rupture after cesarean section in a patient with HELLP syndrome: a case report and review of the literature. Archives of Gynecology and Obstetrics 2007;276(2):189-192.

8. Cernea D. HELLP syndrome complicated with postpartum subcapsular ruptured liver hematoma and putscher-like retinopathy. Case Report. Obstetrics and Gynecology, vol. 2012, Article ID 856135, 4 pages, 2012.

9. Kapana M, Evsenb MS, Gumus M, Onder A, and Tekbas G. Liver hematoma in HELLP syndrome: case report. Gastroenterology Research 2010;3 (3):144-146.

10. Hunter SK, Martin M, Benda JA, Zlantik FJ. "Liver transplant after massive spontaneous hepatic rupture in pregnancy complicated by preeclampsia". Obstet Gynecol 1995; 85: 819_ 22.

11. Ndzengue A, Hammoudeh F, Brutus $P$ et al. An obscure case of hepatic subcapsular hematoma. Case Reports. Gastroenterology 2011;5(1):223-226.

12. Grand'Maison S, Sauve N et al, "Hepatic Rupture in Hemolysis, Elevated Liver Enzymes, Low Platelets Syndrome”. Obstet Gynecol 2012; 119:617-25.

13. Carlson $\mathrm{K}$, Bader $\mathrm{C}$. "Ruptured subcapsular liver hematoma in pregnancy: a case report of nonsurgical management". Am J Obstet Gynecol. 2004; 190:558-60.

14. Pilco $P$ y col. "Hematoma Hepático subcapsular roto en Sind hellp. Revisión de la literatura y reporte de un caso con manejo conservador". Rev. Gastroenterología Perú, 2006; 26: $207-210$.

15. Wicke C. "Subcapsular liver hematoma in HELLP syndrome: evaluation of diagnostic and therapeutic options. A unicenter study". Am J Obstet Gynecol 2004; 190: 106-112.

16. Aldemir M, "Spontaneous liver hematoma and a hepatic rupture in HELLP syndrome: report of two cases". Surg Today 2002; 32: 450-3.

17. Dessole S, Capobianco G, Virdis P, Rubattu G, Cosmi E, and Porcu A. "Hepatic rupture after cesarean section in a patient with HELLP syndrome: a case report and review of the literature," Archives of Gynecology and Obstetrics, vol. 276, no. 2, pp. 189-192, 2007.

18. Stain SC, Woodburn DA, Stephens AL, Katz M, Wagner WH, Donovan AJ. "Spontaneous hepatic haemorrhage associated with pregnancy: treatment by hepatic artery interruption". Ann Surg 1996; 224:72-8.
19. O’Brien J, Buckley O, Munk PL, Torreggiani WC. An unusual case of elevated liver enzymes. Eur Radiol 2007, 17:289-291.

20. Shames BD, Fernandez LA, Sollinger HW, Chin LT, D'Alessandro AM, Knechtle SJ, Lucey MR, Hafez R, Musat AI, Kalayoglu M. Liver transplantation for HELLP syndrome. Liver Transpl 2005, 11(2):224-228.

21. Sibai BM. The HELLP syndrome (Hemolysis, elevated liver enzymes, and low platelets): much ado about nothing?. American Journal of Obstetrics and Gynecology 1990;162(2):311-316.

22. Rinehart BK, Terrone DA, Magann EF, Martin RW, May WL, Martin JN. Preeclampsia-associated hepatic hemorrhage and rupture: mode of management related to maternal and perinatal outcome. Obstet Gynecol Surv 1999; 54:196-202.

23. Stevenson JT, Graham DJ. Hepatic haemorrhage and HELLP syndrome: a surgeon's perspective. Am Surg 1995; 61:756-60.

24. Dimitrios Anyfantakis, Miltiades Kastanakis, Georgios Fragiadakis, Paraskevi Karona, Nikolaos Katsougris, and Emmanouil Bobolakis. Postpartum Spontaneous Subcapsular Hepatic Hematoma Related to Preeclampsia. Hindawi Publishing Corporation, Case Reports in Emergency Medicine, Volume 2014, Article ID 417406, 3 pages.

25. Shaw C, Fattah N, Lynch D, Stokes M. Spontaneous Rupture of The Liver Following a Normal Pregnancy and Delivery. Ir Med J. 2005 Jan; 98 (1): 27-28.

26. Kelly J, Ryan DJ, O'Brien $\mathrm{N}$ and Kirwan WO. Open Access Second trimester hepatic rupture in a 35 year old nulliparous woman with HELLP syndrome: a case report. World Journal of Emergency Surgery 2009, 4:23.

27. Atilla Karateke, Dilek Silfeler, Faruk Karateke, Raziye Kurt, Ayse Guler, and Ismail Kartal. HELLP Syndrome Complicated by Subcapsular Hematoma of Liver: A Case Report and Review of the Literature. Hindawi Publishing Corporation. Case Reports in Obstetrics and Gynecology. Volume 2014, Article ID 585672, 3 pages.

28. Maida Alassia, María Marta Buteler, Cecilia Caffaratti, Hector Bollatti, María Marta Caratti. Hematoma hepático en el síndrome HELLP. Revista de la Facultad de Ciencias Médicas 2011;68(3):119. Córdova, Argentina;

29. Mascarenhas R, Mathias J, Varadarajan R, Geoghegan J and Traynor O. Spontaneous hepatic rupture: a report of five cases”. HPB 2002 ;4 (4): 167-170.

30. Santos-Bolívar J, Perozo-Romero J, Prieto-Montaño J, Reyna-Villasmil E y Torres-Cepeda D. Rotura de hematoma subcapsular hepático como complicación de síndrome de HELLP. Cir Esp 2010; 87 (1): 46 - 58.

31. Pliego A, Zavala J, Porras A. Rotura Hepática espontanea durante el embarazo. Serie de cuatro casos y revisión de la literatura médica. Ginecología y Obstetricia de México 2006; 74: 224-31

32. Quesnel C, Weber A, Mendoza D, Garteiz D. Hematoma hepático espontaneo en embarazo gemelar. Ginecología y Obstetricia de México 2012; 80(2): 110-114.

33. Muñoz C. Hígado graso agudo del embarazo y síndrome HELLP: aspectos diagnósticos y terapéuticos. Gastroenterol Latinoam 2013; Vol 24, Supl No 1: S 123-S 126.

34. Brox-Jiménez A, Ruiz-Luque V,Torres-Arcos C, ParraMembrives P. Experiencia con la técnica de la bolsa de Bogotá para el cierre temporal del abdomen. Cir Esp 2007; 82(3):150-4

35. Pérez L, Pardellas H, Cáceres N. Vacuum assisted closure: utilidad en el abdomen abierto y cierre diferido. Experiencia en 23 pacientes. Cirugía Española 2012; 90(8): 506-512.

\section{ORCID iDs}

Oswaldo M. Gonzales Carrillo

Cristina D. Llanos Torres

Walter Jerry De la Peña Meniz https://orcid.org/0000-0001-9032-1542 https://orcid org/0000-0002-0837-5881 https://orcid.org/0000-0002-3261-773X 